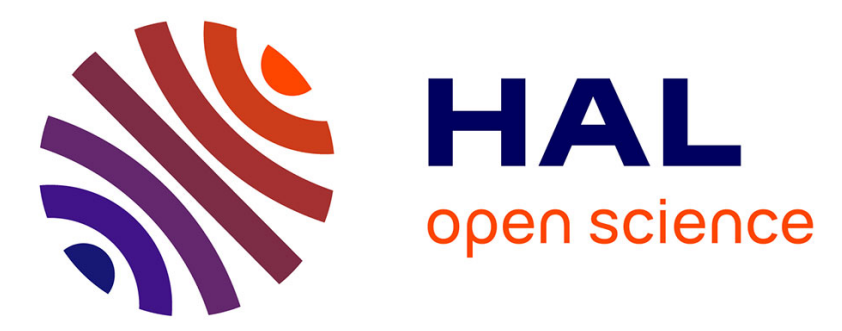

\title{
Plastic heterogeneities of a copper multicrystal deformed in uniaxial tension: experimental study and finite element simulations
}

F. Delaire, Jean L Raphanel, Colette Rey

\section{- To cite this version:}

F. Delaire, Jean L Raphanel, Colette Rey. Plastic heterogeneities of a copper multicrystal deformed in uniaxial tension: experimental study and finite element simulations. Acta Materialia, 2000, 48, pp.1075-1087. 10.1016/S1359-6454(99)00408-5 . hal-00111290

\author{
HAL Id: hal-00111290 \\ https://hal.science/hal-00111290
}

Submitted on 18 Mar 2021

HAL is a multi-disciplinary open access archive for the deposit and dissemination of scientific research documents, whether they are published or not. The documents may come from teaching and research institutions in France or abroad, or from public or private research centers.
L'archive ouverte pluridisciplinaire HAL, est destinée au dépôt et à la diffusion de documents scientifiques de niveau recherche, publiés ou non, émanant des établissements d'enseignement et de recherche français ou étrangers, des laboratoires publics ou privés.

\section{(c)(1)}

Distributed under a Creative Commons Attribution| 4.0 International License 


\title{
PLASTIC HETEROGENEITIES OF A COPPER MULTICRYSTAL DEFORMED IN UNIAXIAL TENSION: EXPERIMENTAL STUDY AND FINITE ELEMENT SIMULATIONS
}

\author{
F. DELAIRE, J. L. RAPHANEL $\dagger \dagger$ and C. REY $\S$ \\ LPMTM-CNRS, Institut Galilée, Université Paris-Nord, Avenue J.-B. Clément, 93430 Villetaneuse,
}

France

\begin{abstract}
A copper sample made of a single layer of grains is plastically deformed by uniaxial tension at room temperature and low strain rate. The deformation field is measured by means of grids deposited on the polished surface of the undeformed specimen and local orientations are recorded using electron back scattering diagrams in a scanning electron microscope. These measures are compared with simulations made by a finite element code using a physically based model for the deformation and hardening of face centered cubic crystals. A good agreement is found between measured and computed values. The simulations give access to much more detail about the history of glide in each grain and help establish which systems are active at a local level. They also provide the evolution of internal variables such as dislocation densities. A new insight into intergranular accommodation as well as intragranular heterogeneities is provided.
\end{abstract}

Résumé: Un échantillon de cuivre constitué d'une seule couche de grains est déformé dans le domaine plastique par traction uniaxiale à température ambiante et à faible vitesse de déformation. Le champ de déformation est mesuré à l'aide de grilles déposées sur la surface polie de l'échantillon non déformé et les orientations locales sont enregistrées par traitement des diagrammes d'électrons rétrodiffusés dans un microscope électronique à balayage. Ces mesures sont comparées à des simulations effectuées avec un code aux éléments finis qui utilise un modèle physique pour la déformation et l'écrouissage des cristaux de structrure cubique à faces centrées. Un bon accord est trouvé entre valeurs mesurées et calculées. Les simulations donnent accès à plus de détails de l'histoire de la déformation par glissement dans chaque grain et aident à établir quels systèmes sont actifs à un niveau local. Elles fournissent aussi l'évolution de variables internes, comme les densités de dislocations. Une nouvelle approche peut ainsi être faite de l'accommodation intergranulaire et de l'hétérogénéité intragranulaire.

Keywords: Mechanical properties; Dislocations; Computer simulation; SEM; Copper

\section{INTRODUCTION}

The plastic response of crystalline materials is in general anisotropic and heterogeneous, which leads to the development of deformation textures and intragranular microstructures. These may be responsible for localization or loss of ductility of the material. It is thus important to gain a better understanding of how an imposed macroscopic

$\dagger$ Present address: LMS, Ecole polytechnique, 91128 Palaiseau Cedex, France.

$\$$ To whom all correspondence should be addressed.

$\S$ Present address: LMSS-MAT, Ecole Centrale Paris, Grande Voie des Vignes, 92295 Châtenay-Malabry Cedex, France. stress or strain is accommodated at the local level of a grain interacting with its immediate neighbors. The many models of crystalline plasticity do not specifically address this matter: the interaction between grains is at best taken in an average sense. For instance, the relaxed Taylor models $[1,2]$ use arguments based on grain shapes and the self-consistent schemes [3-5] place the grain in interaction with a continuum, the equivalent homogeneous medium. This approach is justified since the purpose of these models is the prediction of macroscopic properties, such as deformation textures and overall mechanical response. This class of models includes at their core an averaging scheme in order to allow the transition from the microscale (i.e. the grain scale) to the macroscale. 
The aim of this paper is to present an attempt to calculate by finite element techniques the complete strain and stress fields in aggregates with a small number of grains and to apply the numerical simulation to the case of a copper multicrystal which has been experimentally tested. The use of finite elements in crystalline plasticity has been rapidly growing with the growth of the computing power of the average workstation. Finite element techniques were first used to evaluate the state of stress and strain of bi- and tricrystals [6, 7]. Monotonic, but also cyclic loadings have been investigated [8]. In these papers, however, the overall deformation remained small and no attempt at re-orienting the crystal was performed. Some other finite element codes using crystalline plasticity have aimed directly at describing the polycrystalline behavior, focusing on the development of anisotropy [9], or of deformation textures [10] including, in some instances, temperature and strain rate effects [11]. In most cases, an ideal polycrystal is imagined and relatively few attempts have been made to simulate the behavior of an actual sample [12, 13], one of the reasons being that for a sample with more than one layer of grains, if surface observations provide a lot of detail about the strains and crystal orientation in the surface layer, the boundary conditions imposed by the deeper layers of material cannot be assessed. The problem of number of through thickness grains is addressed in the work by Buchheit et al. [14], but on an ideal polycrystal. Most of these simulations use a hardening rule derived from Asaro and Needleman [15], where quantities similar to critical shear rates are linearly related to glide velocities through a constant hardening matrix which in its simplest form has only two different kinds of term, the diagonal term for self-hardening and the off-diagonal for latent hardening.

The originality of the approach presented here, is to perform the simulation on an actual aggregate made of a single layer of grains and conduct the experiment on the same sample. Moreover, the modeling uses the framework of large deformations and accounts for step by step re-orientation of the crystalline material with a physically motivated hardening rule based on evolution of dislocation densities, so that the hardening matrix is no longer constant but is recalculated and updated at each step.

The special aggregate that we consider shall be called a multicrystal and the material is OFHC copper. It has been possible to grow by the Bridgman technique a multicrystal of about ten grains of millimetric sizes which provided a sample for uniaxial tension made of a single layer of grains with grain boundaries almost orthogonal to the free surfaces. The geometry of the sample and the experimental techniques are described in the next section. The scale of observation and simulation is not that of individual dislocations but rather that of the collective behavior of dislocations or in experimental terms, the scale of the scanning electron microscope (SEM), rather than the transmission electron microscope (TEM). The model and its implementation in the finite element code are detailed in Section 3. One assumes an elasto-viscoplastic behavior of the aggregate, with plastic deformation taking place solely by intragranular dislocation glide on crystallographic slip systems. One attempts to compare quantitatively results of experiments and simulations. The quantities obtained by both methods are the strain tensor (at least some of its components) and the local crystal orientations at predetermined levels of the overall deformation. The simulations are the only means to evaluate the activity of slip systems, the stress field and the dislocation densities. The experimental quantities are computed over a rectangular grid of $1 \times 1 \mathrm{~mm}^{2}$ squares in the undeformed state and the simulated ones at each integration point of the finite element mesh. One shall not only consider the various fields over the sample but also focus at certain selected points in order to illustrate how, within the same grain, different material points undergo different histories of stress and strain and the consequences of these for the heterogeneity of the deformed crystal.

\section{SAMPLE AND EXPERIMENTAL PROCEDURE}

The multicrystal has been taken from an ingot obtained by the Bridgman technique. The sample is made of a thin slice of the ingot, machined so that each surface has almost the same topology and a single layer of grains is present, with grain boundaries almost orthogonal to the free surfaces. The shape of the specimen, with an active length of $29 \mathrm{~mm}$, a width of $16 \mathrm{~mm}$ and a thickness of $3 \mathrm{~mm}$ is chosen so that its ends, which are held by the grips of a tensile machine, do not deform noticeably during the uniaxial tensile test. The specimen is made of a total of 11 grains, with a central grain entirely surrounded by six neighbors (see Fig. 1).

The initial orientations of the grains are determined by the EBSD (electron back scattered diagram) technique performed in a scanning electron

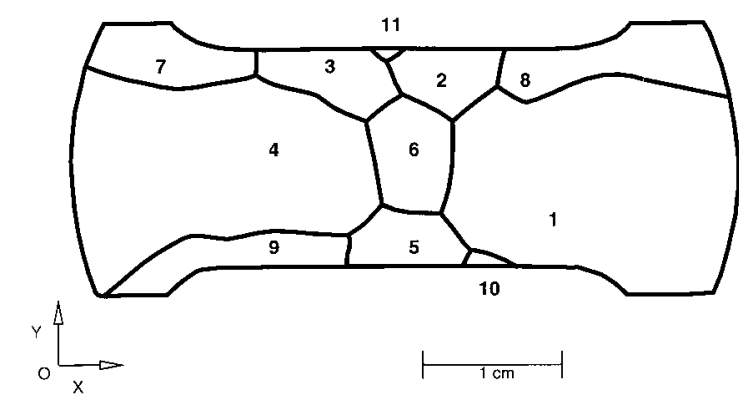

Fig. 1. Sketch of the multicrystal and numbering of its 11 grains. 
microscope (SEM). A microgrid of $1 \times 1 \mathrm{~mm}^{2}$ squares is deposited on one side of the sample. The uniaxial tensile test takes place at constant crosshead velocity of $4 \times 10^{-4} / \mathrm{s}$. The test is stopped at some intervals and the sample observed in the SEM in order to measure local orientations by EBSD and to record the displacements of the nodes of the deformed grid. The slip plane traces are also observed and recorded.

\subsection{Measurements of displacements}

The measurements are performed in a SEM. The in-plane displacements are obtained by the displacement of the stage along two orthogonal axes. The third component is given by a focusing length read on the SEM. The intersections of the grid with the grain boundaries are initially recorded from photographs of the undeformed sample. The position of these points at any later stage, are then estimated by a cubic spline interpolation using the position of the neighboring grid points. A direct measure becomes impossible owing to the contrast values near a grain boundary in the deformed state. The overall precision is $1 \mu \mathrm{m}$ for in-plane displacements and about $50 \mu \mathrm{m}$ for out-of-plane measurements.

\subsection{Strain field computations}

Only six components of the transformation gradient $\mathbf{F}$ are determined by the measured displacements since one associates to a point in the reference configuration its position in the deformed one, namely

$$
M\left(t_{0}\right)=\left(X_{1}, X_{2}\right) \rightarrow M(t)=\left(x_{1}(t), x_{2}(t), x_{3}(t)\right)
$$

so that only the first two columns of $\mathbf{F}$ may be computed, namely

$$
F_{i \alpha}=\frac{\partial x_{i}}{\partial X_{\alpha}}, \quad \text { for } i=1,2,3 \text { and } \alpha=1,2
$$

allowing the computation of three of the Green Lagrange strain tensor components, $E_{11}, E_{22}, E_{33}$, through the well-known relation:

$$
\mathbf{E}=\frac{1}{2}\left(\mathbf{F}^{\mathrm{T}} \mathbf{F}-\mathbf{1}\right)
$$

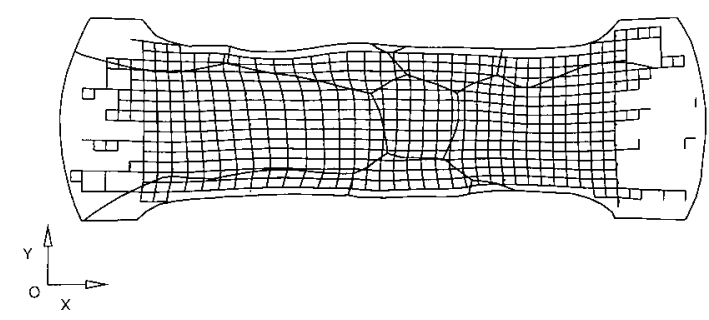

Fig. 2. Deformed sample and grid at 0.30 average axial logarithmic strain.
In order to have an estimate of these strain components at any point of the surface, one may consider the grid of measured points as constituting a mesh in the finite element sense (see Fig. 2), and then use mapping and interpolation functions to compute the values of the components at any spatial location on the sample.

\subsection{Crystalline orientations}

The EBSD technique allows the measurement of local crystalline orientations in the SEM. To any material point in the deformed configuration, one may thus associate a rotation matrix, which allows the passage from the macroscopic reference axes (namely, tensile axis, transverse axis and normal to the plane of the undeformed sample) to the cubic directions in the crystal. In this case also, one may estimate the local orientation at any point by interpolation from the measured ones. For this purpose, the use of the Rodrigues vector [16] is particularly appropriate, since it is defined in a metric space and can provide a measure of the "distance" between two orientations.

\section{MODELING AND SIMULATIONS}

The mechanical modeling follows the framework developed in Teodosiu and co-workers [17]. The main characteristics of the model and its special form that is implemented in the computer code are recalled and outlined in the following subsections.

\subsection{Kinematics and constitutive relations}

The kinematics of large transformations are used which are based on the multiplicative decomposition of the deformation gradient into elastic and plastic parts [18], the velocity gradient $\mathbf{L}$ being subsequently expressed as the sum of an elastic part $\mathbf{L}^{\mathrm{e}}$ and a plastic part $\mathbf{L}^{p}[19,20]$. This requires the choice of intermediate configurations. For crystalline plasticity, the most convenient choice is that of isoclinic, relaxed local configurations [21]. In the case of cubic crystals, one shall simply assume that

Table 1. Schmid and Boas notation

\begin{tabular}{lcc}
\hline Slip system & Plane & Direction \\
\hline A2 & $(\overline{1} 11)$ & {$[0 \overline{1} 1]$} \\
A3 & & {$[101]$} \\
A6 & $(111)$ & {$[110]$} \\
B2 & & {$[0 \overline{1} 1]$} \\
B4 & $(\overline{1} \overline{1} 1)$ & {$[\overline{1} 01]$} \\
B5 & & {$[1 \overline{1} 0]$} \\
C1 & $(1 \overline{1} 1)$ & {$[011]$} \\
C3 & & {$[101]$} \\
C5 & & {$[1 \overline{1} 0]$} \\
D1 & {$[011]$} \\
D4 & & {$[110]$} \\
D6 & & \\
\hline
\end{tabular}


the cubic axes are aligned in the local configurations, with the frame of a Cartesian system of coordinates. For each grain, plasticity is due to glide on crystallographic slip systems. A system $(s)$ is defined by a glide direction $\overline{\mathbf{g}}^{(s)}$ and a glide plane normal $\overline{\mathbf{n}}^{(s)}$ in the isoclinic local configurations. In face centered cubic (f.c.c.) materials such as copper, these planes and directions are well known as the $\{111\}$ planes and $\langle 110\rangle$ directions. There are 12 such slip systems which are referred to by a letter and a number according to the Schmid and Boas notation (see Table 1).

One natural way of coupling the elastic behavior with the flow theory of plasticity is to derive from a linear elastic law an hypoelastic law by time differentiation, so that one may express the time derivative of the Kirchhoff stress $\tau$ in terms of total strain rates, total rotation rates and plastic rate quantities

$$
\dot{\boldsymbol{\tau}}=\mathscr{K}^{\mathrm{e}}: \mathbf{D}+\boldsymbol{\tau} \mathbf{W}-\mathbf{W} \boldsymbol{\tau}-\sum_{s} \dot{\gamma}^{(s)} \mathbf{R}^{(s)}
$$

where

$$
\mathbf{R}^{(s)}=\mathscr{K}^{\mathrm{e}}: \mathbf{D}^{(s)}+\mathbf{W}^{(s)} \boldsymbol{\tau}-\boldsymbol{\tau} \mathbf{W}^{(s)}
$$

with $\mathbf{D}^{(s)}$ and $\mathbf{W}^{(s)}$ being, respectively, the symmetric and antisymmetric parts of $\mathbf{g}^{(s)} \otimes \mathbf{n}^{(s)}$ and $\mathscr{K}^{\mathrm{e}}$ being simply related to the fourth order elasticity tensor (see for instance Ref. [22]).

The rate of slip on an activated slip system is related to the resolved shear by the viscoplastic power law

$$
\dot{\gamma}^{(s)}=\dot{\gamma}_{0}\left|\frac{\tau^{(s)}}{\tau_{\mathrm{C}}^{(s)}}\right|^{n} \operatorname{sgn} \tau^{(s)}
$$

where $\dot{\gamma}_{0}$ is a reference slip rate and the exponent $n$ is of the order of 100 for copper at room temperature. The large value of $n$ implies an almost purely plastic behavior, where $\dot{\gamma}^{(s)}$ remains negligible unless $\left|\tau^{(s)}\right|$ is close to $\tau_{\mathrm{C}}^{(s)}$ which plays the role of a critical shear stress, as in purely plastic models with use of the Schmid law.

\subsection{Hardening law}

One of the advantages of this approach is to use a physically based argument in order to express evolution laws for the critical shears. The more common and more phenomenological approach has been to relate linearly slip rates and critical shear through a hardening matrix with constant coefficients, in general limited to a diagonal one characterizing self-hardening and equal off-diagonal terms associated with latent hardening (see for instance Refs [23-25]). Here, the linear structure of the relation is kept, but one considers that the coefficients of the hardening matrix are not constant and depend on internal variables that are related to the physics of plasticity by dislocation glide.

The model is based on the evolution of dislocation densities [26, 27]. One introduces total dislocation densities on a system $(s), \rho^{(s)}$, whose evolutions are governed by a production term, following Orowan's law and thus linked to a mean free path $L^{(s)}$ and a dynamic recovery term controlled by a critical annihilation length $y_{\mathrm{C}}$. One writes

$$
\dot{\rho}^{(s)}=\frac{1}{b}\left(\frac{1}{L^{(s)}}-2 y_{\mathrm{C}} \rho^{(s)}\right)\left|\dot{\gamma}^{(s)}\right|
$$

where the mean free path is related to the density of point obstacles encountered by the gliding dislocations:

$$
L^{(s)}=K\left(\sum_{u \neq s} \rho^{(u)}\right)^{-1 / 2}
$$

The critical shear stress on a system $(s)$ can be related to the dislocation densities by the hardening relation:

$$
\tau_{\mathrm{C}}^{(s)}=\mu b\left(\sum_{u} a^{(s u)} \rho^{(u)}\right)^{1 / 2}
$$

where $\mu$ is taken as the shear modulus of an isotropic aggregate made of the same material and $a^{(s u)}$ is a matrix taking into account various types of dislocation interactions [28]. The differentiation of this relation with respect to time and some computation lead to the expression of the coefficients of the hardening matrix in terms of the total dislocation densities.

It has been shown by Tabourot et al. [29] that this model can describe the first three stages of the hardening curve of copper single crystals initially oriented for single slip and can also correlate satisfactorily multislip data.

\subsection{Choice of parameters for the model}

The model requires few materials parameters in order to be implemented. Some are well-known values for a material such as copper, others require an identification from curves found in the literature. They fall into the following categories:

$\begin{array}{ll}\text { elastic anisotropy } & \text { the moduli } c_{11}, c_{12}, c_{44} \text { for copper } \\ & \text { single crystals; } \\ \text { viscoplasticity } & \text { a reference glide velocity } \dot{\gamma}_{0} \text {, the } \\ & \text { exponent } n \text { of the viscoplastic power } \\ & \text { law, an initial critical shear } \tau_{\mathrm{C}}^{0} \text {, which } \\ & \text { is the same on all the glide systems } \\ & \text { and shall also be used in order to } \\ & \text { determine an initial dislocation den- } \\ & \text { sity } \rho_{0} ;\end{array}$


hardening

the annihilation distance (for edge dislocations) $y_{\mathrm{C}}$, a material parameter $K$ (needed for the evaluation of the mean free path), the modulus of the Burgers vector $b$, the isotropic shear modulus $\mu$, and the terms of the interaction matrix, $a^{s u}$, which we shall reduce to two values, one for the diagonal terms (self-hardening) and one for the off-diagonal terms (latent hardening).

The values used for our computations are given in Table 2.

\section{FINITE ELEMENT COMPUTATION}

The evolution of the deformation process is described by an updated Lagrangian scheme, that is to say that the configuration at time $t$ is taken as reference configuration for the time interval $[t, t+$ $\Delta t]$. At the end of this interval, the configuration and the internal variables are updated and the new configuration is taken as reference for the next time increment. Let $V$ be the region occupied by the multicrystal at time $t$ and assume that its boundary $\Sigma$ can be composed into two parts $\Sigma_{\tau}$ and $\Sigma_{v}$, such that the traction vector $\mathbf{t}^{*}$ is prescribed on $\Sigma_{\tau}$ and the velocity $\mathbf{v}^{*}$ is prescribed on $\Sigma_{v}$ at any time $t$. In the particular case of uniaxial tension simulations, these conditions become:

- no traction on the free surfaces of the sample;

- imposed velocities at the end surfaces, modeling the grips of the tension machine: one fixed end with zero velocity and the other with a constant axial speed $V_{0}$.

The position vector $\mathbf{x}$, the velocity fields $\mathbf{v}$ and $\delta \mathbf{v}$ are approximated by the same shape functions. The discretization of the kinematical fields leads to the classic discretization of the principle of virtual power that in turn leads to the system of linear algebraic equations:

$$
[\mathbf{K}]\{\Delta \mathbf{u}\}=\{\Delta \mathbf{f}\} .
$$

After solving the system, the configuration and the state variables are updated at each Gauss point by a simple Euler explicit scheme, except for the glide increments that are calculated by the forward gradient approximation [24]. This method presents the drawback to impose very small time increments, but has the advantage of allowing a progressive intragranular lattice reorientaion at each Gauss point so that the incipience of glide on a new slip system at any point of the sample is well accounted for. Attempts at implicit schemes, worked well for single crystals but were less efficient for multicrystals.

The meshing of the structure had the constraint to follow the grain boundaries and also to provide a finer mesh in regions where stronger gradients are expected. The chosen element is a six-node pentahedron, with two integration points. Two layers are used so that there are four integration points through the thickness of the sample (see Fig. 3).

The number of elements has been kept relatively small (469) in order to make the computations in a reasonable time on a powerful workstation. One must compute and update a very large number of variables at each integration point, since in addition to the local orientation (three angles, but in fact a $3 \times 3$ matrix), one updates the dislocation densities on each system (12 quantities) and the stresses. With intermediate quantities used in the computation, it is a total of 134 values per Gauss point that are retained and updated. Another mesh with about the same number of quadratic hexahedral elements has also been tested with very similar numerical results. In order to have a better estimate of quantities in the high gradient regions, one would have to go to the next order of magnitude for the discretization of the structure and consider thousands rather than hundreds of elements. The choice retained here, while partly dictated by the available hardware, was also consistent with the scale of the experimental observations which dealt with millimetric grids deposited on the surface of the sample and manual recording of local orientations.

\section{MAIN FEATURES OF A COMPARISON BETWEEN EXPERIMENT AND SIMULATION}

Three stages of the deformation of the multicrystal have been used for comparing experiment and simulation. They correspond to overall macroscopic axial logarithmic strains of $0.03,0.075$, and 0.33 . At each stage, the sample is placed in the SEM and measurements of grid displacements and local orientations are performed. Three grains are analyzed in detail, which present different patterns of behavior owing to their initial orientations and position within the sample. One has selected the two largest grains near the ends of the sample (grains 1 and 4) and the central grain (grain 6) completely surrounded by others. For these three grains, the initial values of the five largest Schmid factors and the corresponding slip systems are listed in Table 3.

Table 2. Values of the parameters entering the model

\begin{tabular}{lcc}
\hline$c_{11}=166 \times 10^{3} \mathrm{MPa}$ & $c_{12}=120 \times 10^{3} \mathrm{MPa}$ & $c$ \\
$\dot{\gamma}_{0}=10^{-3} / \mathrm{s}$ & $n=100$ & $c_{44}=76 \times 10^{3} \mathrm{MPa}$ \\
$b=2.57 \times 10^{-7} \mathrm{~mm}$ & $y_{\mathrm{C}}=0.5 \times 10^{-6} \mathrm{~mm}$ & $K=75$ \\
isotropic $\mu=45 \times 10^{3} \mathrm{MPa}$ & $a^{s s}=0.52$ & $a^{s u}=0.72, s \neq u$ \\
\hline
\end{tabular}




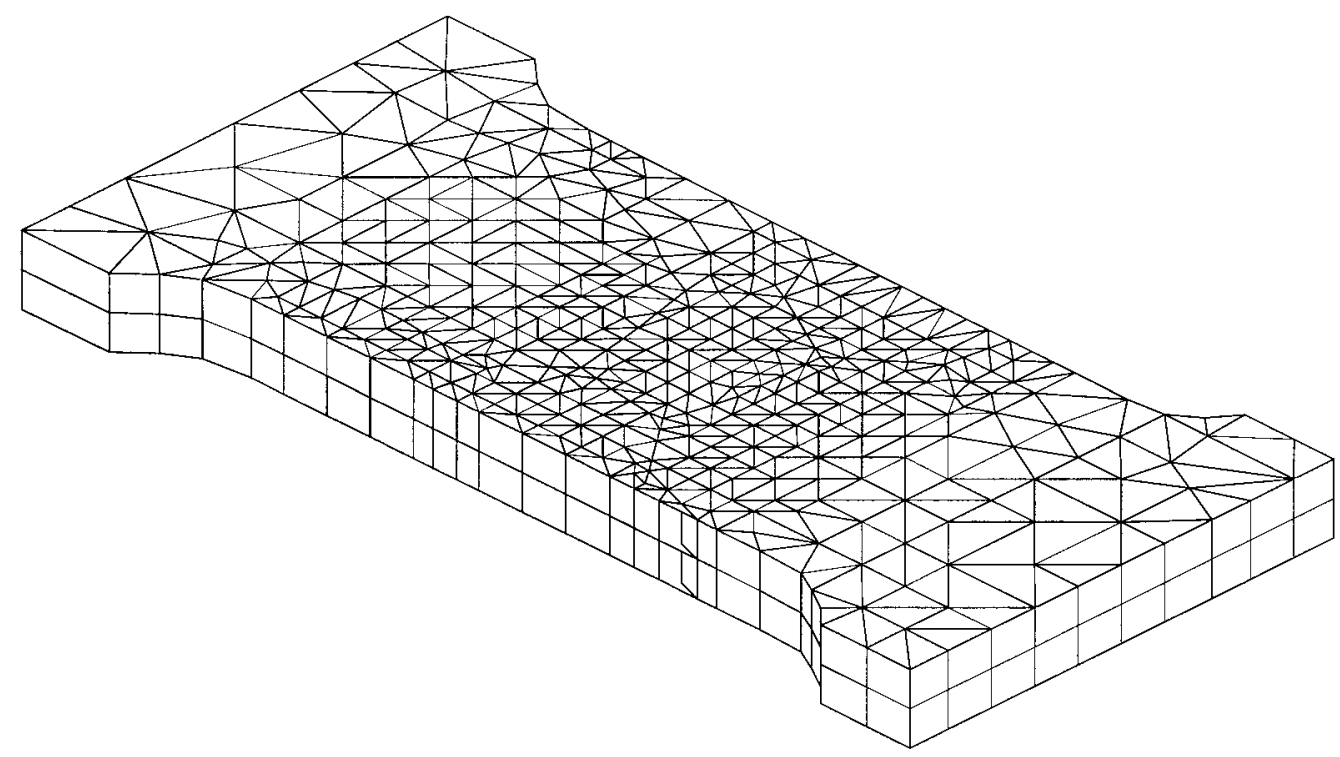

Fig. 3. The finite element mesh: two layers of six-node prismatic elements.

One recalls that the Schmid factor is computed under the assumption of uniaxial tension.

Table 3 indicates that:

- Grain 1 is close to a stable double slip orientation with glide on B4 and C1.

- Grain 4 has an unstable double slip orientation with glide on B4 and A3. The difference between the Schmid factors is however large enough to promote mostly single glide on B4.

- Grain 6 is oriented for single slip on B4.

The actual stress state applied at the boundaries of each grain is not exactly that of a pure tensile test, owing to interactions between grains and actual (or simulated) end conditions. However, the response of the single crystal, initially oriented as the grain in the aggregate can be used as a reference for the discussion. We also point out at this stage that since we deal with a group of crystallites, one cannot replace an orientation by an equivalent one, as in the case of single crystals treated separately. The first grain may be chosen so that B4 is the primary system for uniaxial tension and then the other grains are oriented using the EBSD technique in the SEM, without use of symmetry operations, this procedure ensures that the grain boundaries retain their relative orientation with respect to the grains. A convenient way to represent the initial orien-

Table 3. The five highest Schmid factors of the three grains considered

Grain Schmid factor values and associated glide system

$\begin{array}{llllllllll}0.464 & \text { B4 } & 0.440 & \mathrm{C} 1 & 0.438 & \text { A3 } & 0.414 & \text { A2 } & 0.315 & \text { B2 }\end{array}$

$\begin{array}{llllllllll}0.491 & \text { B4 } & 0.461 & \text { A3 } & 0.308 & \text { B5 } & 0.233 & \text { C1 } & 0.203 & \text { A2 }\end{array}$

$\begin{array}{llllllllll}0.493 & \text { B4 } & 0.439 & \text { A3 } & -0.319 & \text { B5 } & 0.265 & \text { C1 } & 0.211 & \text { A2 }\end{array}$ tations is to draw the inverse pole figure (or its relevant part) which gives the position of the tensile axis with respect to the crystallographic axes (Fig. 4).

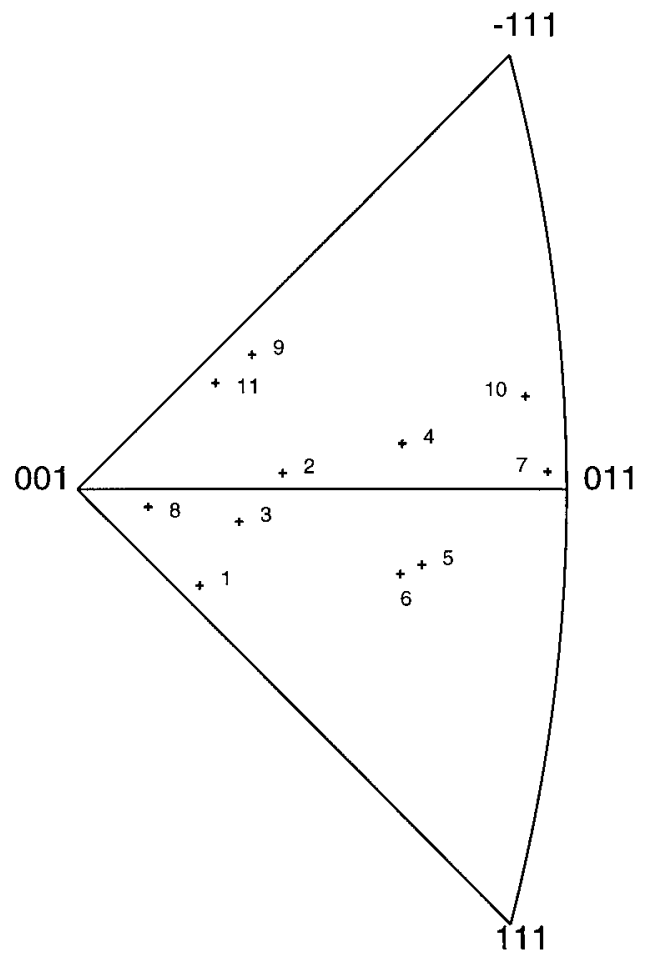

Fig. 4. Inverse pole figure: initial position of the tensile axis with respect to the crystallographic axes for the 11 grains of the sample. 


\subsection{Lagrangian strains and slip system activity}

The plastic deformation of the multicrystal may be studied experimentally by the measure of the surface grid and by the observation of slip plane traces. The computation yields values of the six components of the Lagrangian strain and access to the cumulated glide on every glide system.

5.1.1. Strains. Strain heterogeneities are already present at an average global strain of 0.03. At larger global strains, they remain qualitatively unchanged, even though their extent and magnitude may have varied. Isovalues of axial strain components ignore grain boundaries. The band of localization that goes through grains 9,4 , and 3 is well predicted by the simulation albeit with a lesser spread than in the experiment.

At a global strain of 0.03 , the axial strain $E_{11}$ is already close to 0.06 in a band that stretches across grains 4, 3, and 9. Grain 4 that is in single slip initially deforms a little faster than its neighbors, especially grain 9 which is in symmetric double slip. At larger strains (see Fig. 5), the value of $E_{11}$ in the band becomes two to three times larger than the average value and the band narrows. The exper- imental or simulated axial strain does not show any jump when crossing a grain boundary almost parallel to the tensile axis.

The transverse strain however presents jumps along the grain boundaries parallel to the tensile axis, namely $4-3$ and $4-9$. There is a marked difference between grain 4 which deforms with a very small homogeneous transverse strain and grain 1 which has a negative transverse strain, with a gradient toward and across grain 6 .

5.1.2. Crystallographic slip. A good marker of plastic deformation is provided by the slip plane traces which appear on the polished surface of the sample. Their observation allows in most instances the determination of the corresponding slip system. The ambiguity about glide direction in a given plane is resolved by computation of the Schmid factor in the deformed, i.e. re-oriented state, and in all cases, the experimental data check with the simulations.

The overall picture is given by Fig. 6 which shows the sample at an overall axial strain of 0.075 . The grain boundaries are regions where a strong relief appears, particularly between grains 3 and 4 . The triple points are also regions of fairly high
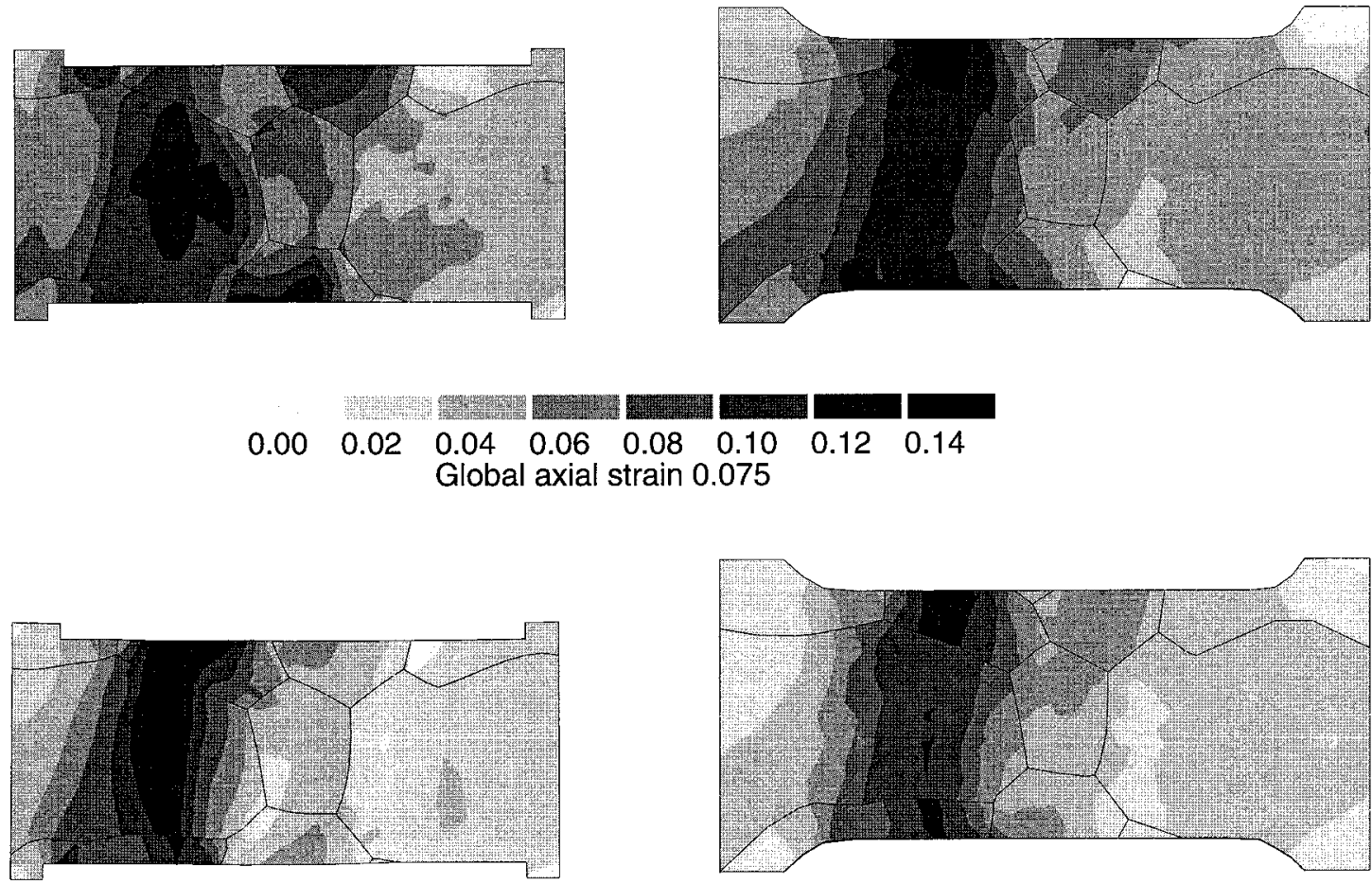

0.00

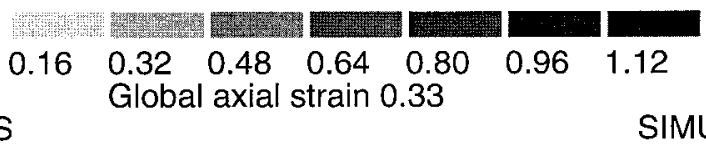

SIMULATIONS

DISTRIBUTIONS OF AXIAL STRAINS E11

Fig. 5. Axial strain distribution. 


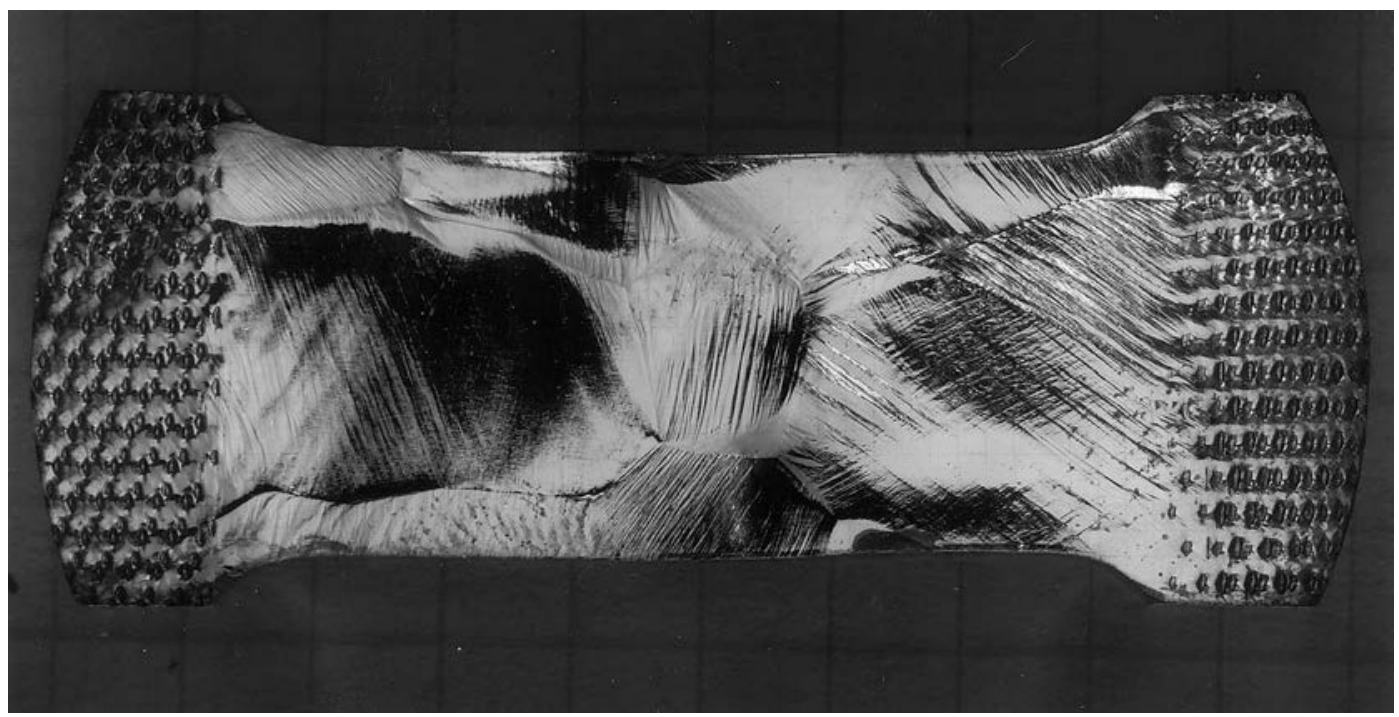

Fig. 6. Picture of the deformed sample at 0.075 average axial strain.

slopes, in particular the junction 1-5-6. In these regions, it is difficult to measure local orientations and strains. The observation of superimposed systems of slip lines in some domains cannot be readily equated with the simultaneous activity of slip systems, but rather tells about a history of plastic deformation. The computed orientations of the slip plane traces are drawn on Fig. 7 for grains 4, 6, and 1 and a direct comparison with Fig. 6 shows the close agreement. Figure 8 is a sketch deduced from Fig. 6 that outlines the intragranular domains with different slip activity.

5.1.2.1. Grain 1. Slip line patterns limit four intragranular domains. A multiple slip domain in a central position where the two expected systems are active together and three other regions where the interactions with the neighboring grain boundary (GB) or triple point play a part: a region with mostly $\mathrm{C} 1$ activity, along the boundary with grain
8; a deep region stretching from the border with grain 2 with B4 activity and a local zone of A3 near the GB; a region with a sharp relief near the triple point $6-5-1$.

Computed glide quantities agree with these observations and give an insight into the development of plastic deformation in the different intragranular regions. For a given material point and a given slip system, the plot of the glide quantity with respect to the macroscopic axial strain (which plays the part of a time increment) allows us to follow the local plastic activation of the different slip systems as the overall deformation proceeds. One has selected four points for grain 1 which are representative of the different behaviors (see Fig. 9). There is a good agreement between the observed slip plane traces and the most active slip plane, either $\mathrm{B} 4$ or $\mathrm{C} 1$ depending on the region considered. One notices that near GB $1-2$ and GB $1-6$, the primary system B4 stops gliding and single glide on $\mathrm{C} 1$ then
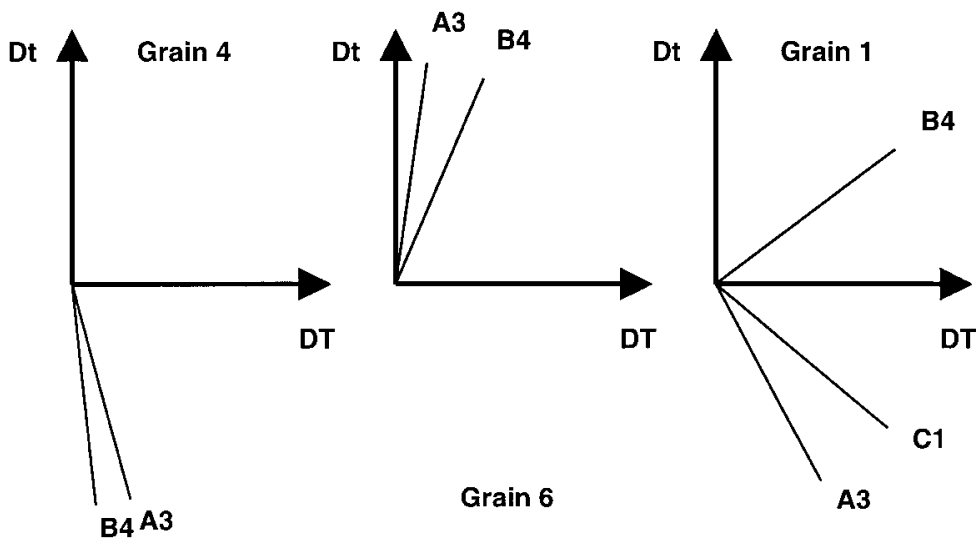

Fig. 7. Computed orientation of the slip plane traces for grains 1, 4, and 6 at about 0.07 average axial strain. 


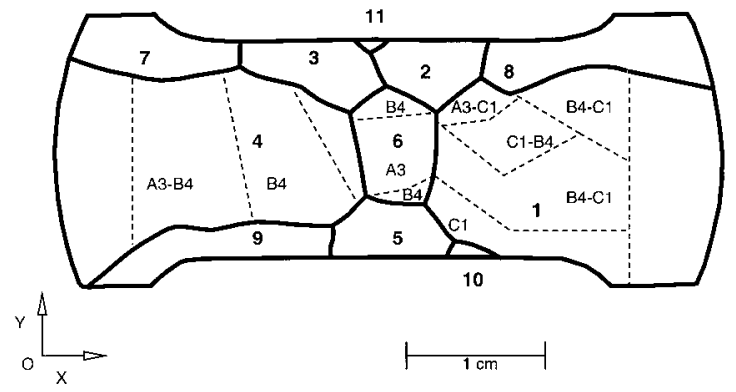

Fig. 8. Domains of different glide activity for grains 1, 4, and 6.

appears. On the contrary it is a situation of multiple slip on $\mathrm{B} 4$ and $\mathrm{C} 1$ that takes place near GB $1-8$ and at larger strains near GB 1-10.

5.1.2.2. Grain 4. This grain is characterized by large axial deformation taking place in a band not far from the boundary with grain 6 and which also extends into its upper and lower neighbors. One can distinguish three zones: a peripheral zone, GB 4-7, 4-3, 4-6, and 4-5 where few slip lines are observed and a strong relief is present, a large domain of mostly B4 activity and a wide band stemming from GB 4-9 where traces of critical system $\mathrm{A} 3$ are observed.

The simulations (see Fig. 10) however indicate that along the grain boundaries as well as in the grain interior single glide B4 prevails and indeed a band where glide is fairly large appears, just next to the boundary with grain 6 , in a section where one finds grains 9-4-3 in "parallel". A closer analysis of the simulation shows that the axial deformation starts to increase or "localize" in grain 9 which has a large initial Schmid factor and is oriented for stable double slip and that subsequently, grain 4 deforms very rapidly. It appears that systems A3 (critical or secondary system) and B5 (tertiary system) play a role in accommodating the conditions imposed by the boundary. The cumulated glides on these two systems remain small but may be critical to ensure compatibility.

5.1.2.3. Grain 6. In this central grain, one may distinguish four regions, namely the top of the grain, the boundary with grain 4 , the bottom of the grain and the center region with an extension to the boundary with grain 1 . In the first three regions, one notices mostly single slip, although the sharp
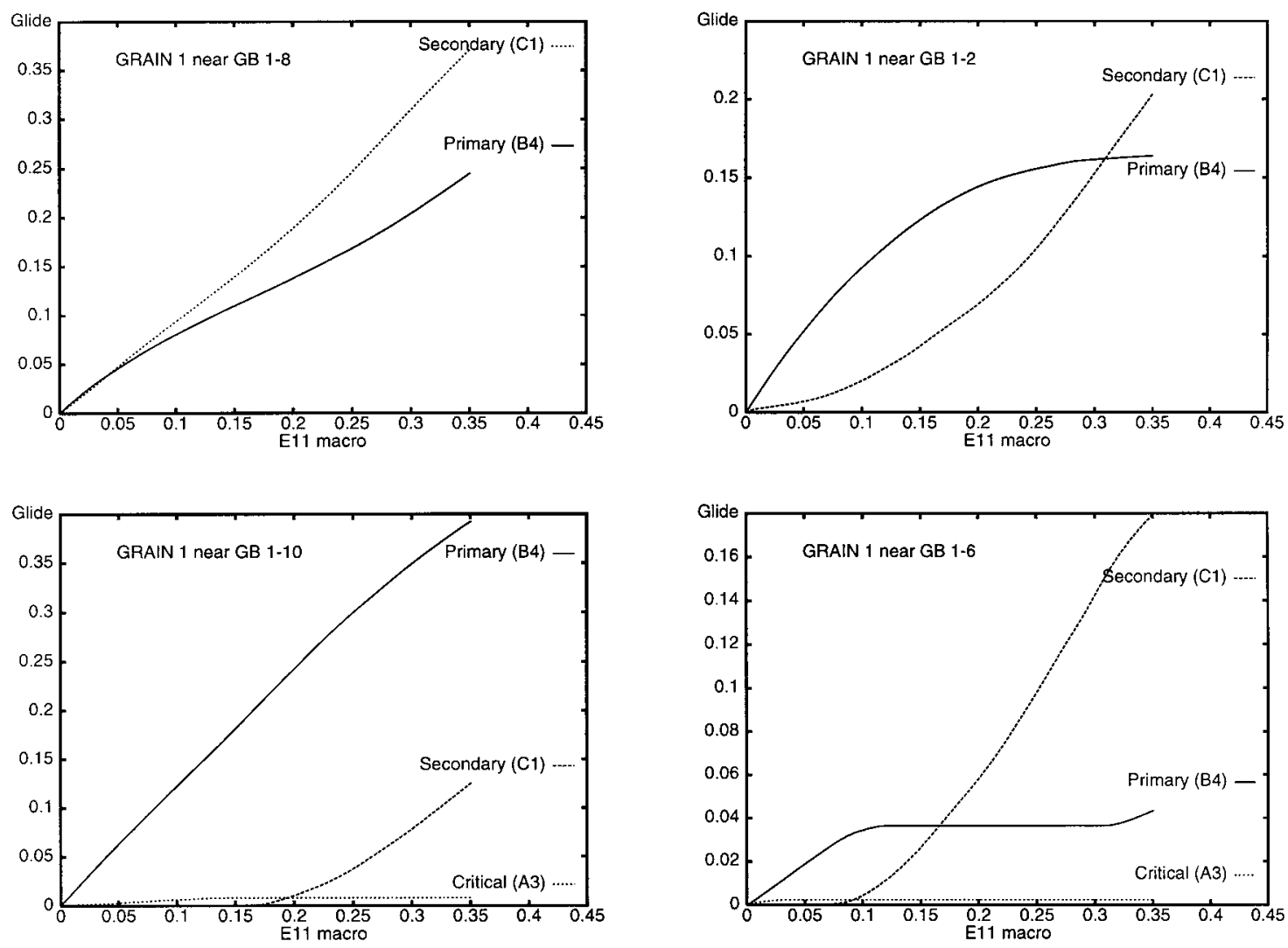

Fig. 9. Glide activity at four different points of grain 1 . 

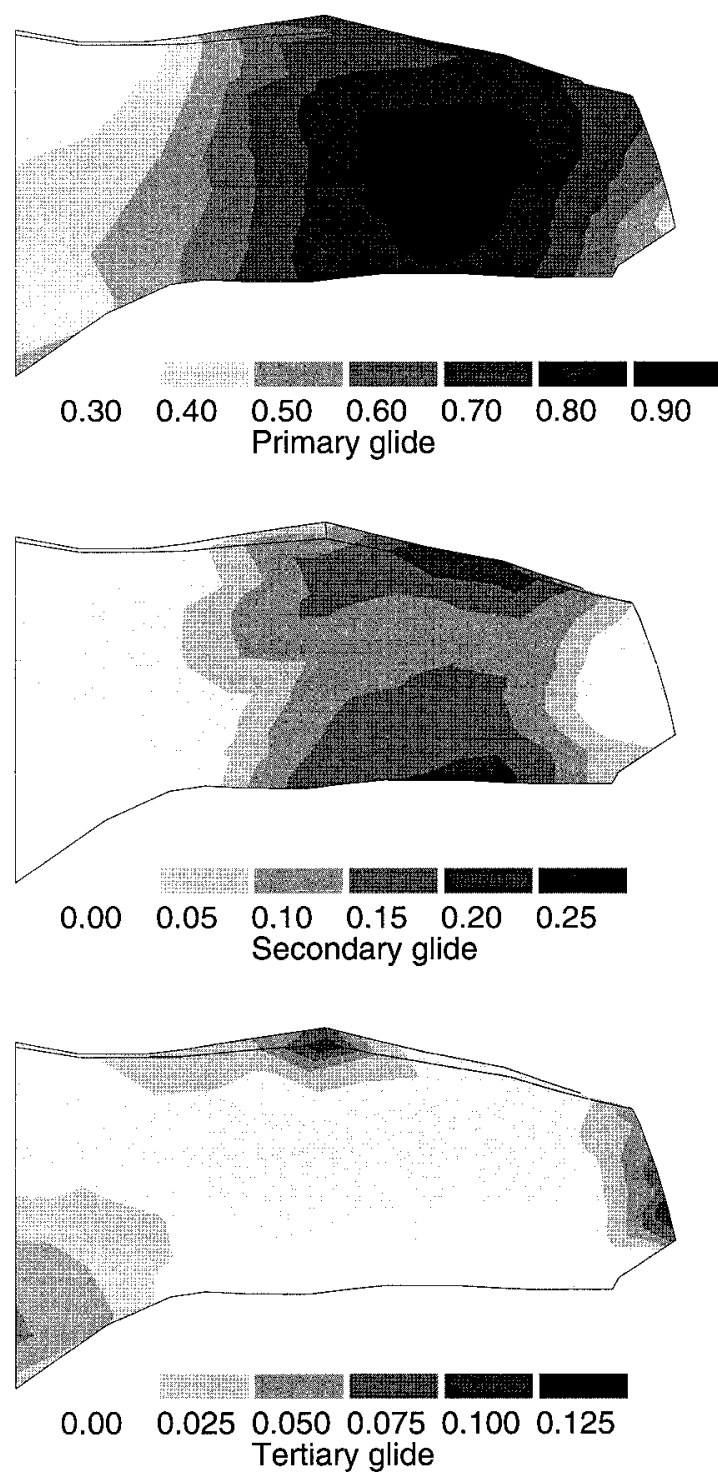

Fig. 10. Slip activity in grain 4 at the overall axial strain of 0.33 .

relief near the GB makes precise observations difficult. In the more central region, A3 and B4 are present.

The computations of glide quantities at several locations within grain 6 support these observations and allow the analysis of the sequence of events leading to the observed slip plane traces. The interior of the grain starts in double slip A3-B4, for axial strains up to 0.1 , when the activity of $\mathrm{B} 4$ stops and single glide A3 takes place. It is interesting to notice that inside grain 1 , we have observed the same situation near the middle of the GB 1-6, where glide on B4 stops for $E_{11}=0.1$ and glide on the secondary system $\mathrm{C} 1$ appears. But for grain 1, plastic glide had initiated on B4 only.

\subsection{Orientations}

The inverse pole figures (Fig. 11) show a good agreement between experiment and simulation at least for the intermediate global axial strain of 0.075 .

For the central grain however, there is more dispersion in the measured orientations than expected by the simulation. One should emphasize that the experimental points are surface measurements, since the electron beam interacts with an area of about $7 \mu \mathrm{m}^{2}$ and a depth of $10-50 \mathrm{~nm}$ while the computed orientations are evaluated at the Gauss points of the mesh which are at least $300 \mu \mathrm{m}$ below the surface. The grain that presents the largest re-orientation is grain 4 , which is the most deformed, with a band of localization of axial deformation. It is also a grain that deforms mostly by single slip. As expected, most of the grain rotates towards a "stable" orientation for uniaxial tension, namely $\langle 112\rangle$ for the tensile axis as one can see on the corresponding inverse pole figure. The same tendency is found for grain 1. Not as many points are available for grain 6, experimentally or in the simulations, and for this grain the agreement is the least satisfying. Owing to the more complex state of interaction between neighbors and the relatively smaller size of grain 6, the exact spatial position of the point of measure or of computation may be of greater importance here, and one also notices that this is a grain which presents quite important out of plane deformation, near triple points and boundaries.

\subsection{Dislocation densities}

The dislocation densities are the internal variables of our plasticity model. One should of course exert a certain caution in the discussion of the results and keep in mind that in this respect there may exist a discrepancy between the actual physical dislocation density and what the computations yield. In particular, we have chosen rather arbitrarily an initial value and a rather phenomenological way of accounting for dislocation density evolutions. It appears, however, that at least qualitatively, the evolutions of the computed quantities may be linked to evolutions of dislocation densities. The total dislocation density remains fairly homogeneous through the grains, and in this sense does not show maxima in the band of localized axial deformation. It thus appears that two grains such as grains 1 and 4 present very different axial strains and glide activities but have almost the same total dislocation density (see Fig. 12). It may now be interesting to look at the dislocation density on the primary glide system. There, again, for the two grains 1 and 4, we find fairly similar values and this can be understood by analyzing the evolution law for dislocation densities, and more particularly, the 


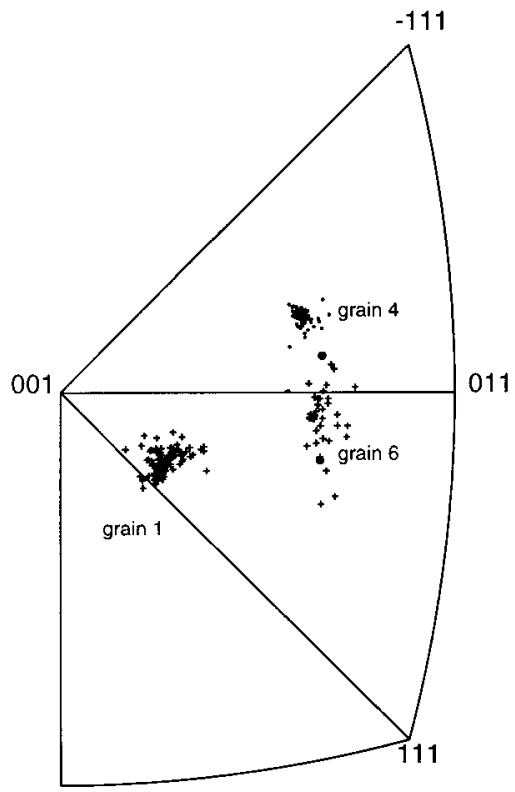

EXPERIMENTS

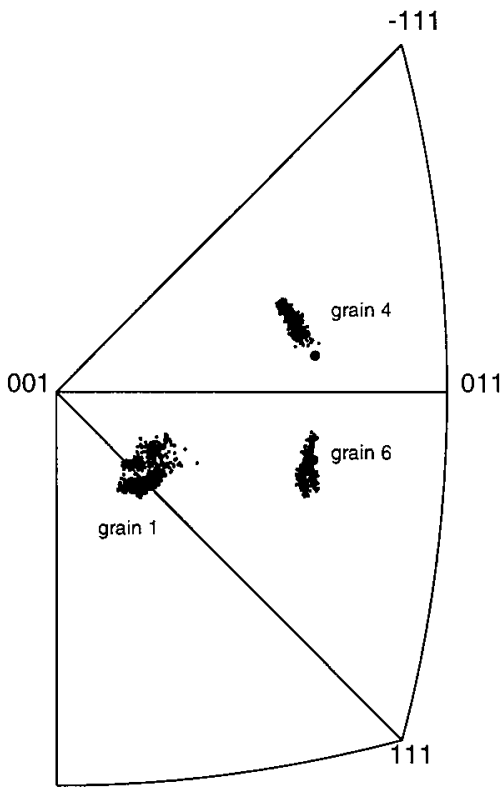

SIMULATIONS

Fig. 11. Inverse pole figures (tensile axis) for grains 1, 4, and 6 at an axial strain of 0.075 (the larger dot represents the initial orientation, see also Fig. 4).

evolution of the mean free path. Grain 4 is for the most part of its strain history, in single slip, the mean free path associated with the primary system does not change. On the contrary, grain 1 is almost always in double slip, so that the mean free path associated with these systems decreases almost like the square root of the total dislocation density, hence reducing the corresponding glide quantities.

\section{CONCLUSION}

The multicrystal, at an intermediate scale between the single crystal and the polycrystal, is the first relatively complex structure that yields both to experimental and numerical studies, in order to provide a better understanding of the development of plastic heterogeneities in crystalline materials. The comparisons between observation, measurements and simulations have shown a good overall agreement of similar quantities, namely strain and local orientations. The kinetics of plastic glide has also been accurately described, with no discrepancy between methods for the determination of locally active slip systems. The experimental and the numerical scales have been chosen of the same order, that is, slightly less than a millimeter. Since the grains are mostly centimetric, this constitutes the first smaller order necessary for a description of intragranular heterogeneities.

A first striking result is the analysis of the devel- opment of a zone of strain concentration which crosses three grains of the multicrystal. The simulations show that the phenomenon starts at the beginning of the plastic stage, too early to be measured by the grid. At first, a grain along the edge (grain 9) is well oriented for stable double slip and deforms easily. It favors somewhat the deformation of its immediate neighbor (grain 4) which is oriented for single slip and rotates toward a stable orientation. The band of strain concentration presents a maximum that reaches three times the global axial strain and ignores grain boundaries aligned with the tensile axis.

Another important result concerns grain interactions and this can be seen very clearly for grain 6 which is surrounded with neighbors and may thus be the grain less likely to behave like a homogeneous single crystal in uniaxial tension. Grain 6 presents a fairly large gradient of re-orientation, with a maximum near the boundary with grain 4 . In grain 6, one observes also gradients of axial, transverse and shear components of strain. It is placed "in series" between two large grains, grains 1 and 4, which behave very differently, as can be seen not only in terms of magnitude but also of shape of the local strain tensors and it must also accommodate "in parallel" grains 3 and 2 on one side and grain 5 on the other. This accommodation does not seem to be achieved by a layer at the periphery 

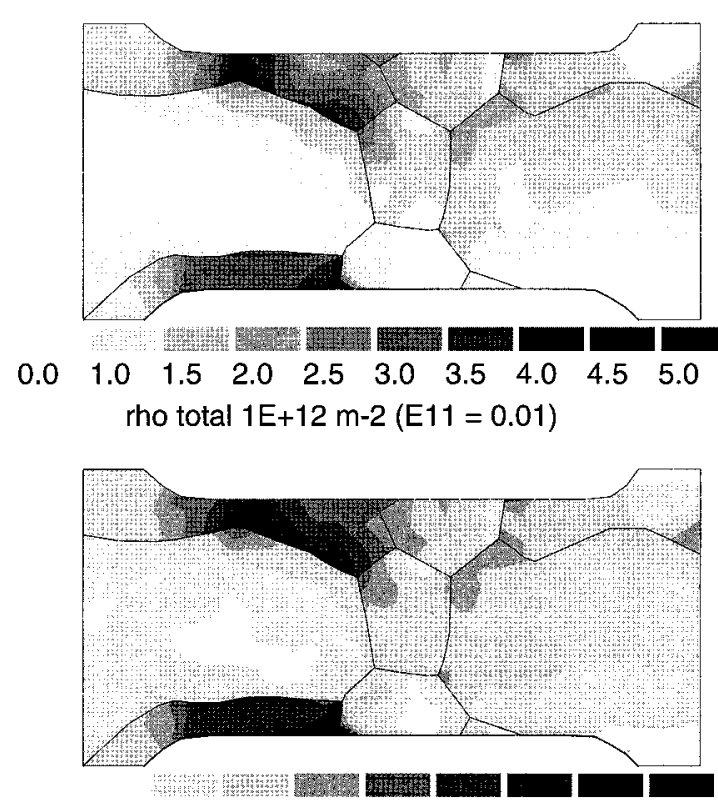

$\begin{array}{lllllllll}0.0 & 0.25 & 0.5 & 0.75 & 1.0 & 1.25 & 1.5 & 1.75 & 2.0\end{array}$ rho total $1 E+13 \mathrm{~m}-2(\mathrm{E} 11=0.075)$

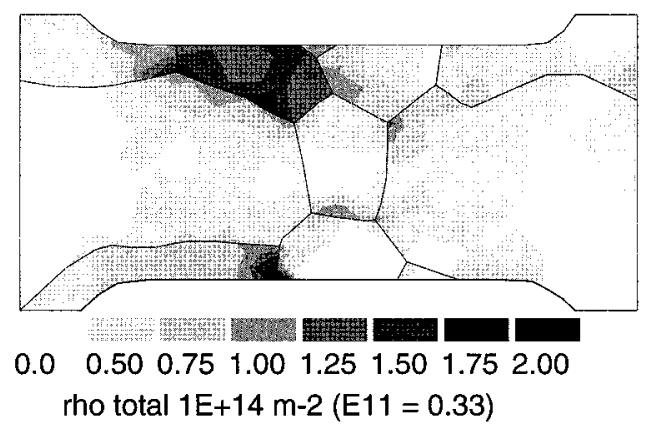

Fig. 12. Total dislocation densities.

of the grain, with the inside of the grain behaving somewhat homogeneously. One tends to see intragranular bands extending from a grain boundary and often bounded by lines originating at a triple point as a virtual extension of a grain boundary.

These results were made possible through the combination of the experimental and numerical techniques. The mesh that has been used however limits the accuracy of our analysis. The use of a different mesh of the same characteristic coarseness, with two layers of quadratic hexahedral elements, does not change dramatically the simulated values, except for stresses that are more accurately estimated. This shows that significant improvement requires to go at least one order of magnitude higher for mesh refinement, with the corollary that experimental data should also be gathered at a smaller scale. Unfortunately, multicrystals are diffi- cult to produce and even more difficult to reproduce so that one cannot multiply experiments.

A critical point of the model remains the hardening laws. They are very material, temperature, rate and grain size dependent. Without changing the framework presented here, one could envision introducing a local mean free path, bounded by a distance to the nearest grain boundary, measured along a glide direction. This would introduce more clearly a characteristic length and grain size dependence. It would also be of interest to vary hardening parameters in order to better assess the influence of each hardening mechanism, but this would constitute a whole study by itself, albeit a study made possible by the numerical tool which has been presented here.

\section{REFERENCES}

1. Kocks, U. F. and Canova, G. R., in 2nd RISØ Symposium: Deformation, Mechanisms, Microstructure, Roskilde, Denmark, ed. N. Hansen et al, 1981, pp. 35-44.

2. van Houtte, P., Mater. Sci. Engng, 1982, 55, 69.

3. Hill, R., J. Mech. Phys. Solids, 1965, 13, 89.

4. Molinari, A., Canova, G. R. and Ahzi, S., Acta metall., 1987, 35, 2983.

5. Zaoui, A., Int. J. Solids Struct., 1972, 8, 1089.

6. Ohashi, T., Trans. Japan Inst. Metals, 1987, 28, 906.

7. Havliček, F., Kratochvil, J., Tokuda, M. and Lev, V., Int. J. Plasticity, 1990, 6, 281.

8. Méric, L., Cailletaud, G. and Gaspérini, M., Acta metall. mater., 1994, 42, 921.

9. Takahashi, H., Motohashi, H., Tokuda, M. and Abe, T., Int. J. Plasticity, 1994, 10, 63.

10. Mathur, K. K. and Dawson, P. R., Int. J. Plasticity, $1989, \mathbf{5}, 67$

11. Khotari, M. and Anand, L., J. Mech. Phys. Solids, 1998, 46(1), 51

12. Becker, R. and Richmond, O., Modelling Simul. Mater. Sci. Engng, 1994, 2(3A), 439.

13. Becker, R. and Panchanadeeswaran, S., Acta metall. mater., 1995, 43(7), 2701.

14. Buchheit, T. E., Bourcier, R. J., Wellman, G. W. and Neilsen, M. K., Modelling Simul. Mater. Sci. Engng, 1997, 5, 421.

15. Asaro, R. J. and Needleman, A., Acta metall., 1985, 33, 923 .

16. Becker, R. and Panchanadeeswaran, S., Textures Microstruct., 1989, 10, 167.

17. Raphanel, J. L., Teodosiu, C. and Tabourot, L., Finite element simulation of the large elastoplastic deformation of multicrystals, in Large Plastic Deformations: Fundamental Aspects and Applications to Metal Forming, ed. C. Teodosiu, J. L. Raphanel and F. Sidoroff. A. A. Balkema, Rotterdam, 1993, pp. 153-168.

18. Lee, E. H., J. appl. Mech., 1969, 36, 1.

19. Rice, J. R., J. Mech. Phys. Solids, 1971, 19, 433.

20. Mandel, J., Plasticité classique et viscoplasticité, in Lecture Notes CISM, Udine 1971. Springer-Verlag, Berlin, 1972.

21. Teodosiu, C. and Sidoroff, F., Int. J. Engng Sci., 1976, 14, 165.

22. Peirce, D., Asaro, R. J. and Needleman, A., Acta metall., 1982, 30, 1087.

23. Asaro, R. J., Adv. appl. Mech., 1983, 23, 1.

24. Peirce, D., Asaro, R. J. and Needleman, A., Acta metall., 1983, 31, 1951. 
25. Needleman, A., Asaro, R. J., Lemonds, J. and Peirce, D., Comput. Methods appl. Mech. Engng, 1985, 52, 689. 26. Teodosiu, C., in NBS Spec Pub. 317, Vol. 2, 1970, pp. 837-876.

27. Kröner, E. and Teodosiu, C., in Problems of
Plasticity, ed. A. Sawczuk. Noordhoff International, Leyden, 1972, pp. 49-82.

28. Franciosi, P., Acta metall., 1985, 33, 1601.

29. Tabourot, L., Fivel, M. and Rauch, E., Mater. Sci. Engng, 1997, A234-236, 639. 